\title{
Neutral theory exposes the challenge of bending the curve of biodiversity loss for the post-2020 Global Biodiversity Framework
}

\author{
Falko Buschke ${ }^{1}$ \\ ${ }^{1}$ University of the Free State
}

March 18, 2021

\begin{abstract}
In May, nations of the world will meet to negotiate the post-2020 Global Biodiversity Framework under the Convention on Biological Diversity. An influential ambition is "bending the curve of biodiversity loss", which aims to reverse the decline of global biodiversity indicators. A second relevant, yet less prominent, milestone is the 20th anniversary of the publication of The Unified Neutral Theory of Biodiversity and Biogeography. Here, I apply neutral theory to show how global biodiversity indicators for population size (Living Planet Index) and extinction threat (Red List Index) decline under neutral ecological drift. This demonstrates that declining indicators alone do not necessarily reflect deterministic species-specific or geographical patterns of biodiversity loss. Thus, "bending the curve" could be assessed relative to a counterfactual based on neutral theory, rather than static baselines. If used correctly, the 20-year legacy of neutral theory can be extended to make a valuable contribution to the post-2020 Global Biodiversity Framework
\end{abstract}

Neutral theory exposes the challenge of bending the curve of biodiversity loss for the post-2020 Global Biodiversity Framework

Falko. T Buschke ${ }^{1,2 *}$

Centre for Environmental Management (IB 67), University of the Free State, P.O. Box 339, Bloemfontein, South Africa

Animal Ecology, Global Change and Sustainable Development, KU Leuven, Leuven, Belgium

* falko.buschke@gmail.com

Key words: Biodiversity indicators, Convention on Biological Diversity, counterfactuals, extinction, Living Planet Index, Neutral Theory, Red List Index

Running Head: Neutral theory and the post-2020 Global Biodiversity Framework

Type of submission : Viewpoint

Word count: 1,697 words

Abstract: 152 words

Number of figures: 2

Number of tables: 0

Number of references: 18 references

Supporting code: All code used in the submission is available from https:/github.com/falkobuschke/NeutralGBF and will be deposited to a permanent repository (Figshare) upon acceptance. 


\begin{abstract}
In May, nations of the world will meet to negotiate the post-2020 Global Biodiversity Framework under the Convention on Biological Diversity. An influential ambition is "bending the curve of biodiversity loss", which aims to reverse the decline of global biodiversity indicators. A second relevant, yet less prominent, milestone is the $20^{\text {th }}$ anniversary of the publication of The Unified Neutral Theory of Biodiversity and Biogeography. Here, I apply neutral theory to show how global biodiversity indicators for population size (Living Planet Index ) and extinction threat (Red List Index) decline under neutral ecological drift. This demonstrates that declining indicators alone do not necessarily reflect deterministic species-specific or geographical patterns of biodiversity loss. Thus, "bending the curve" could be assessed relative to a counterfactual based on neutral theory, rather than static baselines. If used correctly, the 20-year legacy of neutral theory can be extended to make a valuable contribution to the post-2020 Global Biodiversity Framework
\end{abstract}

Key words: Biodiversity indicators, Convention on Biological Diversity, counterfactuals, extinction, Living Planet Index, Neutral Theory, Red List Index

In May, nations of the world will meet in Kunming, China, where they will negotiate a post-2020 Global Biodiversity Framework under the Convention on Biological Diversity. The overall failure to meet global biodiversity targets during the previous decade (IPBES 2019, Secretariat of the Convention on Biological Diversity 2020) has raised the stakes for these negotiations. One prominent ambition is "bending the curve of biodiversity loss" (Mace et al. 2018; Díaz et al. 2020; Leclère et al. 2020) because the next three decades should not only stop the downward trajectories of population sizes and extinction threats, but also redirect these upwards.

Although "bending the curve" is an engaging visual metaphor for the post-2020 Global Biodiversity Framework, it needs to be made tractable by science-based biodiversity targets and indicators. The global indicators being put forward in Appendix 1 by the Open-ended Working Group on the post-2020 Global Biodiversity Framework (2020) necessarily reduce uncountable biological complexity into simple metrics. These indicators include the Living Planet Index, a global indicator of vertebrate population trends (Collen et al. 2009; WWF 2020); and the IUCN Red List (IUCN 2012) and its associated Red List Index(Butchart et al. 2004), global indicators of incremental extinction threat. These indicators were integral to the global assessment of the Intergovernmental Science-Policy Platform for Biodiversity and Ecosystem Services (IPBES 2019) as well as the Global Biodiversity Outlook Report 5 under the Convention on Biological Diversity (Secretariat of the Convention on Biological Diversity 2020). Moreover, population trends and species extinctions were included in the pioneering efforts to develop global pathways and mitigation scenarios for biodiversity (Leclère et al. 2020). These indicators define the curve that needs to be bent by mid-century and it is tempting to interpret downward or upward trends in these indicators as signs of human impact or conservation effectiveness, respectively. But is this interpretation always true?

It is not enough that indicators rise or fall with underlying biodiversity variables; upward or downward trends should also be attributable to real biological changes rather than random dynamics. This point is largely ignored in global biodiversity monitoring frameworks, which tend to interpret indicators relative to static baselines. However, as the Global Biodiversity Framework dominates our collective attention, this year also marks a second, less conspicuous, milestone: the 20th anniversary of the publication of The United Neutral Theory of Biodiversity and Biogeography(Hubbell 2001). In the following sections, I posit that neutral theory provides valuable insights into the way global biodiversity indicators behave under random dynamics. Neutral theory has established a controversial legacy over the last two decades by showing how simple stochastic births, deaths, speciation and migration can predict many patterns in nature. The controversy stems from neutral theory's assumption that individual organisms are demographically equivalent (i.e. neutral), even though this assumption is obviously false (Hubbell 2001; Rosindell et al. 2011; Leroi et al. 2020). Nevertheless, neutral theory answers the question of what biodiversity patterns would look like if individuals of different species were interchangeable. Often, such neutral patterns are indistinguishable from empirical data; much to the chagrin of those studying the nuanced natural histories of different species. Therefore, neutral theory could serve as a valuable null model for global biodiversity indicators. 
Here, I will use the simplest possible model based on neutral theory to illustrate how global biodiversity indicators behave in the absence of any deterministic species-specific threats. It would be convenient if these indicators were stable in the absence of deterministic species-level trends, but, as I demonstrate in the subsequence sections, this is not the case. I specifically present the most basic neutral model for two reasons. First, I hope to portray neutral theory in a way that is accessible to non-specialists, particularly the policymakers working towards the post-2020 framework. Second, I want to demonstrate that even the coarsest neutral approximations can have heuristic value for global biodiversity policy. All code needed to replicate this model is included as Supplementary Files.

The neutral model considers a saturated community of $J=5000$ individuals from $S=40$ species across 50 years between 1970 and 2020 . The community is closed to migration and speciation rates are zero (although these processes can be included in more complex neutral models using the parameters $m$ and $\nu$, respectively: Hubbell 2001). At the start of the simulation in 1970, the Jindividuals are randomly assigned to the $S$ species. In every subsequent year, all individuals die and are replaced (i.e. zero-sum dynamics), but the relative probability that a replacement belongs to a specific species is proportional to that species' relative abundance in the preceding year. In ecological terms, this could be imagined as a community of annual plants without overlapping generations or a long-lived seedbank. Each year, all individual plants die after producing a fixed number of seeds regardless of their species identity, so that the structure of the plant community in the next year depends how many seeds were produced in the year before. As the years pass, the effect of random deaths and births accumulate so that some populations become more common, while others gradually decline. Such random fluctuations of species abundance are known as ecological drift (Hubbell 2001).

Populations in a neutral community fluctuate randomly under ecological drift (Fig. 1a). Population sizes, $N$, are equally likely to increase or decrease between the two fixation points of extinction $(N=0)$ or monodominance $(N=J)$, yet random ecological drift results in a declining Living Planet Index (Fig. 1b). Although one expects the Living Planet Index to remain stable in the absence of deterministic trends, the neutral model shows how ecological drift can cause the index to decline by as much as $20 \%$ in 50 years, even though the total number of individual organisms is constant (Fig. 1). The Living Planet Index declines due to ecological drift because it is based on year-on-year changes in populations measured as $\lambda=\log \left(\mathrm{N}_{\mathrm{t}+1} / \mathrm{N}_{\mathrm{t}}\right)$ (Collen et al. 2009; WWF 2020). This formulation was designed for exponential population growth, where doubling a population is symmetrical to halving the same population, even though the absolute change in the population is twice as much in the former. By contrast, upward and downward ecological drift are equally likely, but the log-transformation ensures that positive fluctuations cannot compensate for negative fluctuations, hence a declining index.

The same neutral model can be used to explore indicators of extinction threat. In the absence of dispersal or speciation, all species in neutral communities will eventually drift to extinction, except for one random species that becomes mono-dominant. So, neutral simulation can be iterated to estimate how species' abundances affect their extinction rates (Fig. 2a). The IUCN Red List defines three levels of extinction threat (IUCN 2012): critically endangered (CR: 50\% extinction probability within 10 years), endangered (EN: $20 \%$ extinction probability within 20 years), or vulnerable (VU: $10 \%$ extinction probability within 100 years). So, I allowed neutral populations to fluctuate randomly and calculated how long it took them to drift to extinction (Fig. 2a). Using 10,000 iterations of the neutral model, I measured how often species went extinct across three time-intervals (10,20 and 100 years) for every increment of species abundance. This allowed me to quantify how ecological drift caused species to transition between Red List categories (Fig. $2 \mathrm{~b}$ ) and then calculate the proportion of threatened species in the community through time (Fig. 2c). This adds context to calls for defining a headline global conservation priority based on extinction (Rounsevell et al . 2020), because it implies that indicators of extinction risk, such as the Red List Index, can gradually worsen due to random chance alone (Fig. 2d).

Declining biodiversity indicators will have policy ramifications if conservation scientists attribute these patterns to human pressures. But the simple neutral model presented here shows how the Living Planet Index 
and the Red List Index decline even in the absence of threatening processes or disproportionate sensitivity of certain species. This is significant considering Goal A2 in the zero draft of the post-2020 Global Biodiversity Framework, which proposes that "The number of species that are threatened is reduced by [X\%] and the abundance of species has increased on average by [X\%]" (Open-ended Working Group on the post-2020 Global Biodiversity Framework 2020). Although the exact percentages are yet to be negotiated at the Congress of Parties, these percentages should ideally distinguish between deterministic declines in biodiversity indicators and declines that are indistinguishable from random ecological dynamics.

By this point, critics will be crying out that biodiversity loss is obviously non-neutral. There is considerable evidence that population declines, and extinction risks vary across taxonomic groups and biogeographical regions (Hilbers et al. 2017; Leung et al.2020; WWF 2020). However, the purpose of neutral theory is not to explain biodiversity patterns, but rather to predict what patterns would look like if species were equivalent (Rosindell et al. 2011; Leroi et al. 2020). Neutral theory can be used to model multiple biodiversity targets simultaneously even in the absence of species- or threat-specific empirical data. Thus, it can contribute to agenda-setting and target formulation or applied retrospectively during target review ( $c f r$. Nicholson et al. 2019). Furthermore, even though the model presented here was purposely simplistic, simulations can be made more sophisticated by adding dispersal and speciation. Adjusting these parameters have, for example, already been be used to predict extinction debt following habitat fragmentation (Thompson et al. 2019) or whether restoration can mitigate human impacts (Buschke \& Sinclair 2019).

If used correctly, the 20-year legacy of neutral theory can be extended to make a valuable contribution to the post-2020 Global Biodiversity Framework. This contribution will not be about showing how empirical biodiversity trends are due to random chance alone. Instead, neutral theory could be used to model counterfactuals against which empirical trends can be compared (Nicholson et al . 2019). Comparing empirical patterns to neutral simulations will allow us to pin-point whether sensitive species contribute disproportionately to indicator declines (e.g. Leung et al . 2020), or whether declines in one geographical region consistently differ from those in another region, given natural differences in species richness and abundance. Therefore, neutral theory provides the essential context needed to accurately measure global biodiversity ambitions towards "bending the curve of biodiversity loss" (Mace et al . 2018; Díaz et al. 2020; Leclère et al. 2020).

\section{Acknowledgements}

I thank Ryan Chisholm and James Rosindell for commenting on an earlier version of this manuscript.

\section{References}

Buschke, F.T. \& Sinclair, S.P. (2019). Adding ecological and evolutionary processes to restoration biodiversity offset models using neutral theory. Divers Distrib , 25, 1351-1361.

Butchart, S.H.M., Stattersfield, A.J., Bennun, L.A., Shutes, S.M., Akçakaya, H.R., Baillie, J.E.M., et al. (2004). Measuring Global Trends in the Status of Biodiversity: Red List Indices for Birds.PLoS Biol , 2 , e383.

Collen, B., Loh, J., Whitmee, S., McRae, L., Amin, R. \& Baillie, J.E.M. (2009). Monitoring change in vertebrate abundance: the Living Planet Index. Conserv Biol , 23, 317-327.

Hilbers, J.P., Santini, L., Visconti, P., Schipper, A.M., Pinto, C., Rondinini, C., et al. (2017). Setting population targets for mammals using body mass as a predictor of population persistence. Conserv Biol , 31, $385-393$.

Hubbell, S.P. (2001). The unified neutral theory of biodiversity and biogeography . Princeton University Press, Princeton, NJ.

IPBES. (2019). Summary for policymakers of the global assessment report on biodiversity and ecosystem services of the Intergovernmental Science-Policy Platform on Biodiversity and Ecosystem Services. IPBES secretariat, Bonn, Germany. 
IUCN. (2012). IUCN Red List Categories and Criteria: Version 3.1 . Second edition. International Union for the Conservation of Nature, Gland, Switzerland.

Leclère, D., Obersteiner, M., Barrett, M., Butchart, S.H.M., Chaudhary, A., De Palma, A., et al. (2020). Bending the curve of terrestrial biodiversity needs an integrated strategy. Nature , 585, 551-556.

Leroi, A.M., Lambert, B., Rosindell, J., Zhang, X. \& Kokkoris, G.D. (2020). Neutral syndrome. Nat Hum Behav , 4, 780-790.

Leung, B., Hargreaves, A.L., Greenberg, D.A., McGill, B., Dornelas, M. \& Freeman, R. (2020). Clustered versus catastrophic global vertebrate declines. Nature, 58, 267-271.

Mace, G.M., Barrett, M., Burgess, N.D., Cornell, S.E., Freeman, R., Grooten, M., et al. (2018). Aiming higher to bend the curve of biodiversity loss. Nat Sustain , 1, 448-451.

Nicholson, E., Fulton, E.A., Brooks, T.M., Blanchard, R., Leadley, P.et al. (2019). Scenarios and models to support global conservation targets. Trends Ecol Evol, 34, 57-68.

Open-ended Working Group on the post-2020 Global Biodiversity Framework. (2020). Zero draft of the post-2020 Global Biodiversity Framework(No. CBD/ WG2020/2/3). Convention on Biological Diversity.

Rosindell, J., Hubbell, S.P. \& Etienne, R.S. (2011). The Unified Neutral Theory of Biodiversity and Biogeography at age ten. Trends Ecol Evol , 26, 340-348.

Rounsevell, M.D.A., Harfoot, M., Harrison, P.A., Newbold, T., Gregory, R.D. \& Mace, G.M. (2020). A biodiversity target based on species extinctions. Science, 368, 1193-1195.

Secretariat of the Convention on Biological Diversity. (2020). Global Biodiversity Outlook 5 . Convention on Biological Diversity, Montreal.

Thompson, S.E.D., Chisholm, R.A. \& Rosindell, J. (2019). Characterising extinction debt following habitat fragmentation using neutral theory.Ecol Lett , 22, 2087-2096.

WWF. (2020). Living Planet Report 2020 - Bending the curve of biodiversity loss. World Wide Fund for Nature, Gland, Switzerland.

\section{Figure Captions}

Fig. 1: The Living Planet Index for a neutral community.(a) Zero-sum e cological drift of $J=5000$ individuals across $S=40$ species between 1970 and 2020.(b) The mean and $95 \%$ confidence intervals of Living Planet Index (LPI) for the neutral community, where the baseline is set to population abundance in 1970.

Fig. 2: The extinction risk of a neutral community.(a) Extinction probability as a function of abundance across three time intervals $(10,20$ and 100 years) for a neutral community of $J=5000$. Coloured lines show the thresholds for critically endangered (CR), endangered (EN) and vulnerable (VU) populations.(b) The zero-sum ecological drift of $J=5000$ individuals from $S=40$ species between 1970 and 2020, superimposed with coloured thresholds of extinction threat categories.(c) The percentage of each species per threat category between 1970 and 2020, and (d) the associated Red List Index (where values of 1 indicate all species are Least Concern, LC, and 0 indicates that all species are extinct, EX).

\section{Figure 1}



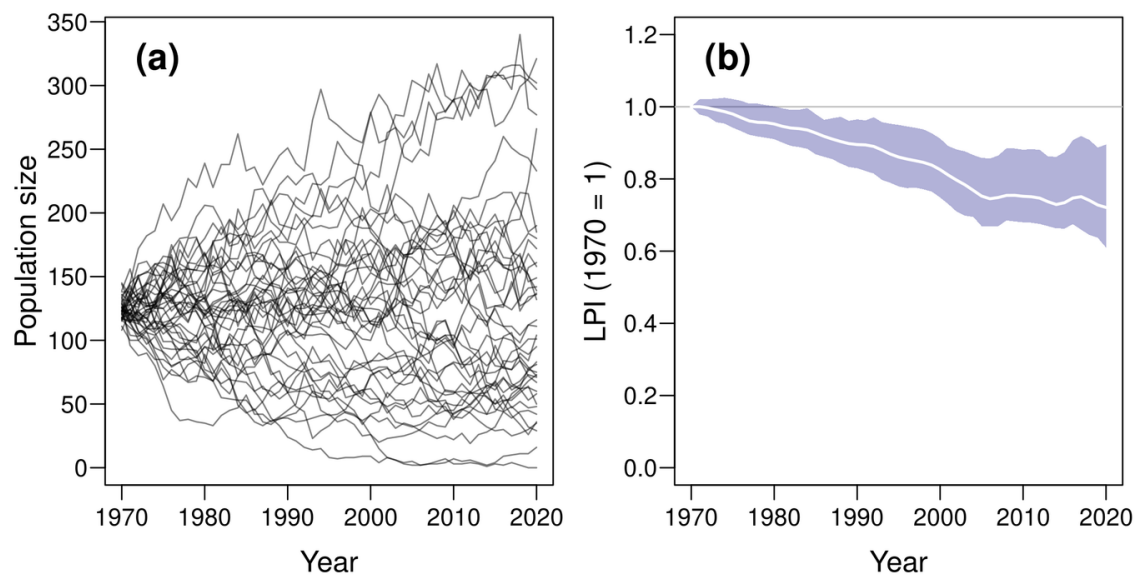

Fig. 1: The Living Planet Index for a neutral community.(a) Zero-sum e cological drift of $J=5000$ individuals across $S=40$ species between 1970 and 2020.(b) The mean and $95 \%$ confidence intervals of Living Planet Index (LPI) for the neutral community, where the baseline is set to population abundance in 1970.

Figure 2
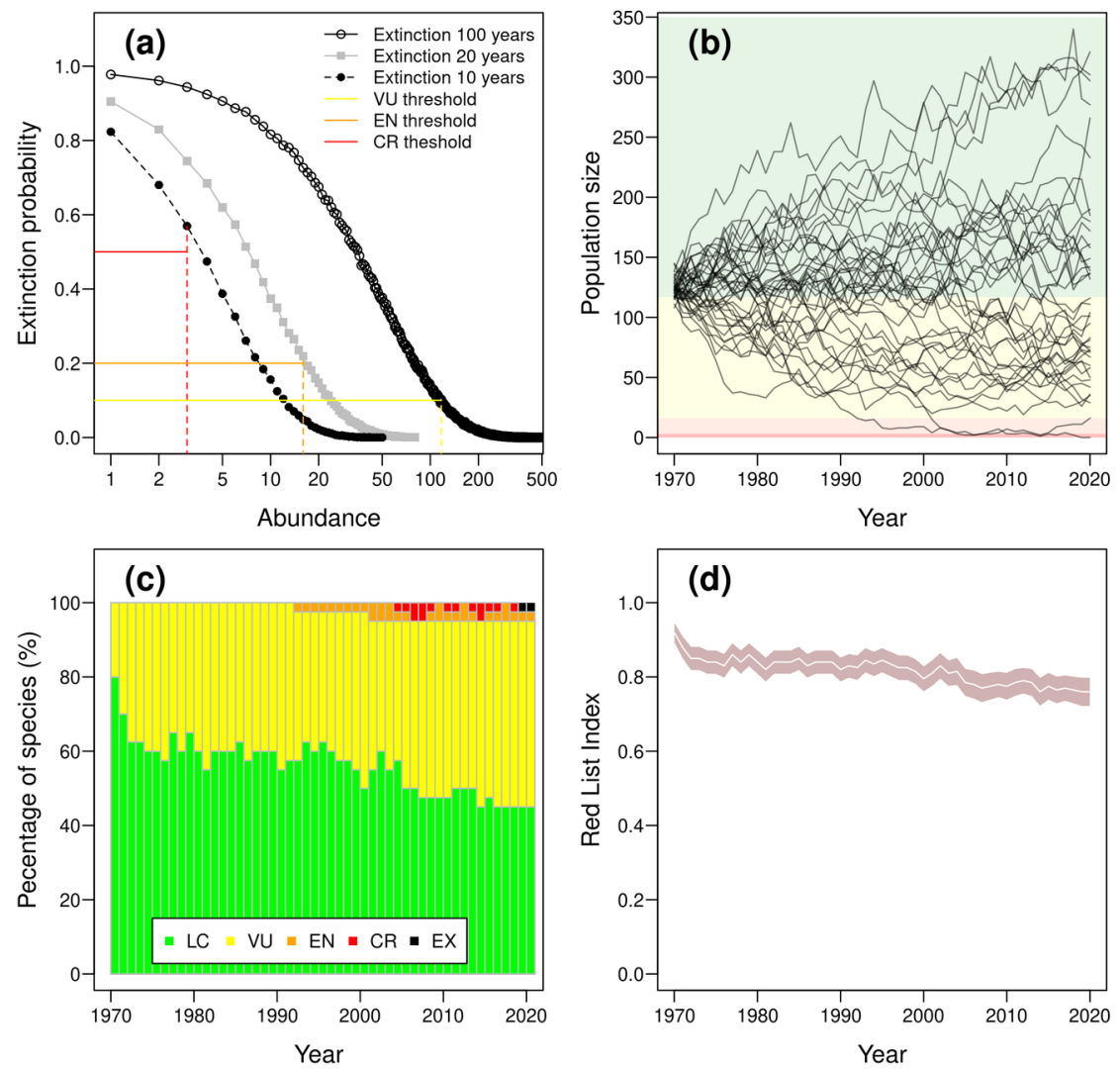

Fig. 2: The extinction risk of a neutral community.(a) Extinction probability as a function of abundance across three time intervals (10,20 and 100 years) for a neutral community of $J=5000$. Coloured lines show 
the thresholds for critically endangered (CR), endangered (EN) and vulnerable (VU) populations.(b) The zero-sum ecological drift of $J=5000$ individuals from $S=40$ species between 1970 and 2020, superimposed with coloured thresholds of extinction threat categories.(c) The percentage of each species per threat category between 1970 and 2020, and (d) the associated Red List Index (where values of 1 indicate all species are Least Concern, LC, and 0 indicates that all species are extinct, EX). 